\title{
Patient-centered outcomes: a bridge too far?
}

\author{
Barbara B Mittleman and Peter E Lipsky
}

Recent emphasis on patient-centered outcomes in the rheumatology community has shifted the goals of therapy from remission of disease activity to the return of wellness. While a laudable goal, this shift toward patient-centered outcomes might represent a 'bridge too far' in the clinical management of patients with antirheumatic therapy, and could present a challenging barrier in clinical trials.

An implicit conundrum is presented by the so-called patient-centered outcomes-are the outcomes related to disease or to illness? Although disease certainly relates to illness, disease is "something an organ has", whereas "illness is something a man (sic) has" (DeBusk RF et al. (1999) Arch Intern Med 159: 27392742). Thus, illness can be understood as the subjective experience of disease, whereas disease is the pathologic process causing tissue or organ damage. Each is distinct although they are closely intertwined; common physiologic pathways are involved in both. Clearly there can be a great deal of disease with little illness (i.e. occult malignancy) and considerable illness with minimal disease.

In general, patient-centered outcomes such as quality of life assessments measure the impact of an intervention on illness. If illness is a surrogate for disease, this might be appropriate. Illness, however, also includes psychological, sensory, affective, cognitive, cultural and spiritual considerations, and therefore, frequently does not accurately parallel disease.

At a time when most antirheumatic therapy was empiric and not very effective, patientcentered outcomes served an important role in assessing the effect of therapy. Feeling better probably meant doing better, even if disease was changed very little. More recently, highly active targeted antirheumatic therapies have become available. These agents specifically ameliorate aspects of disease. It is neither obvious nor predictable that reducing disease will necessarily have a positive impact on illness. Insofar as tumor necrosis factor (TNF) bridges disease ...specific

approaches

aimed at

the illness

component

of rheumatic

disease might

be developed,

improving

our ability to

provide more

comprehensive

care

$B B$ Mittleman is the Director of the Program on Public-

Private Partnerships,

Office of Science

Policy, NIH, Bethesda, $M D, U S A$, and PE

Lipsky is the Editor-

in-Chief of Nature

Clinical Practice

Rheumatology.

\section{Competing interests}

The authors declared

they have no competing

interests.

www.nature.com/clinicalpractice doi:10.1038/ncprheum0289 and illness in patients with rheumatoid arthritis, we might expect that anti-TNF therapy would improve both illness and disease. However, we know neither the role of TNF in rheumatic disease, nor how rheumatoid inflammation affects illness behavior or perception. In fact, we know that inflammation only accounts for a fraction of measurable disability in rheumatoid arthritis (Scott DL et al. (2000) Rheumatology (Oxford) 39: 122-132), whereas co-existent fibromyalgia might account for considerable disability in systemic lupus erythematosus (Middleton GD et al. (1994) Arthritis Rheum 37: 1181-1188).

Clinical trial design, therefore, must address features of disease and features of illness separately and explicitly. Only by developing sensitive and objective measures of pathobiology can the extent and nature of an agent's action on the disease process be accurately evaluated. Such outcome measures relate directly to the effect of targeted therapy on the disease process and directly measure an agent's effectiveness. These data can inform the use and development of agents that might be revolutionary in their ability to prevent or forestall disease processes leading to disability, deformity and even mortality.

Similarly, the development of sensitive instruments to assess features and characteristics of disease-associated illness will permit both evaluation of how an intervention affects patientcentered outcomes, and enhance our understanding of how disease and illness interrelate in the setting of antirheumatic therapy. Indeed, from this analysis, specific approaches aimed at the illness component of rheumatic disease might be developed, improving our ability to provide more comprehensive care. Although this research agenda is important and deserves more attention, patient-centered outcomes cannot substitute for pathobiologic information to judge efficacy and effectiveness of targeted antirheumatic therapy - not until both the relation of disease and illness, and the specific role of the targeted molecules or pathways in that equation are better characterized. 